\title{
Discriminación por morfometría geométrica de once especies de Anopheles (Nyssorhynchus) presentes en Colombia
}

\author{
David Alonso Calle ${ }^{1}$, Martha Lucía Quiñones ${ }^{2}$, Holmes Francisco Erazo ${ }^{3}$, Nicolás Jaramillo ${ }^{1}$ \\ 1 Grupo de Chagas, Instituto de Biología, Universidad de Antioquia, Medellín, Colombia \\ 2 Facultad de Medicina, Universidad Nacional de Colombia, Bogotá, D.C. Colombia \\ 3 Departamento Administrativo de Salud de Putumayo, Mocoa, Colombia \\ El trabajo fue realizado en el Laboratorio de Chagas de la Universidad de Antioquia.
}

Introducción. Es importante determinar las especies de Anopheles pertenecientes al subgénero Nyssorhynchus, dado que allí se encuentran los vectores principales de malaria en Colombia.

Objetivo. Utilizar la morfometría geométrica para evaluar la variación morfométrica de once especies del subgénero Nyssorhynchus, presentes en Colombia y la capacidad de diferenciar las hembras.

Materiales y métodos. Los especímenes fueron obtenidos de mosquitos silvestres, series e isofamilias de hembras recolectadas en cebos humanos protegidos y fueron identificados en todos sus estadios asociados usando las claves convencionales. A partir de fotografías digitales del ala derecha de 336 ejemplares de las 11 especies se seleccionaron 12 puntos anatómicos. Los puntos se convirtieron en coordenadas, las cuales se procesaron mediante el análisis generalizado de Procrustes. Se obtuvieron variables de tamaño y conformación, las cuales fueron analizadas mediante estadísticas univariadas y multivariadas.

Resultados. Las técnicas de morfometría geométrica demuestran que la división en secciones del subgénero Nyssorrhynchus no se correlaciona con la conformación de las alas y demostraron que sólo con 12 puntos anatómicos se logra diferenciar en sus respectivas especies el $97 \%$ de los especímenes de la sección Argyritarsis y el $86 \%$ de la sección Albimanus del subgénero Nyssorhynchus, de forma cuantitativa y sin ambigüedades. Además, se separaron los individuos de tres especies que habitan en simpatría en el Putumayo, difíciles de discriminar en el estadio de adulto hembra.

Conclusión. La mayoría de especies de Anopheles (Nyssorhynchus) presentes en Colombia se pueden diferenciar al utilizar la morfometría geométrica como herramienta de apoyo a las claves convencionales.

Palabras clave: Anopheles/anatomía e histología, clasificación, vectores de enfermedades, malaria, Colombia.

Differentiation by geometric morphometrics among 11 Anopheles (Nyssorhynchus) in Colombia.

Introduction. The correct identification of the Anopheles species of the subgenus Nyssorhynchus is important because this subgenus includes the main malaria vectors in Colombia. This information is necessary for focusing a malaria control program.

Objective. Geometric morphometrics were used to evaluate morphometric variation of 11 species of subgenus Nyssorhynchus present in Colombia and to distinguish females of each species. Materials and methods. The specimens were obtained from series and family broods from females collected with protected human hosts as attractants. The field collected specimens and their progeny were identified at each of the associated stages by conventional keys. For some species, wild females were used. Landmarks were selected on wings from digital pictures from 336 individuals, and digitized with coordinates. The coordinate matrix was processed by generalized Procrustes analysis which generated size and shape variables, free of nonbiological variation. Size and shape variables were analyzed by univariate and multivariate statistics. 
Results. The subdivision of subgenus Nyssorhynchus in sections is not correlated with wing shape. Discriminant analyses correctly classified $97 \%$ of females in the section Albimanus and $86 \%$ in the section Argyritarsis. In addition, these methodologies allowed the correct identification of 3 sympatric species from Putumayo which have been difficult to identify in the adult female stage.

Conclusion. The geometric morphometrics were demonstrated to be a very useful tool as an adjunct to taxonomy of females the use of this method is recommended in studies of the subgenus Nyssorhynchus in Colombia.

Keywords: Systematics, Nyssorhynchus, Section Argyritarsis, Section Albimanus, geometric morphometrics, malaria vectors.

La malaria humana constituye la principal causa de morbilidad y mortalidad en los países tropicales, donde, aproximadamente, $2 \times 10^{9}$ de personas están expuestas a contraer la enfermedad (1). Anualmente se reportan a escala mundial entre 300 y 500 millones de casos (1).

En su mayoría, los vectores en esta región pertenecen al subgénero Nyssorhynchus, y los principales son: Anopheles (Nyssorhynchus) darlingi Root, A. (Nys.) albimanus Wiedemann y A. (Nys.) nuneztovari Gabaldón (2), aunque los estudios recientes han señalado otras especies de este subgénero como posibles transmisores de la enfermedad (3). Es importante diferenciar las especies vectoras de aquéllas que no tienen un papel importante en la transmisión con el fin de dirigir adecuadamente las medidas de control acordes a su comportamiento, biología, respuesta a la presión de insecticidas y otras características que son propias de cada especie.

Las claves existentes para la determinación taxonómica de las hembras de las especies del subgénero Nyssorhynchus (4-7) no permiten una correcta determinación taxonómica entre algunas especies y esto es un problema cuando sólo es posible tener acceso a las hembras y éstas se encuentran en simpatría. Tal es el caso de las especies de $A$. (Nys.) nuneztovari y A. (Nys.) rangeli Gabaldón, Cova García \& López, o A. (Nys.) oswaldoi (Peryassu), A. (Nys.) benarrochi

\footnotetext{
Correspondencia:

Nicolás Jaramillo, Calle 62 № 52-59, sexto piso, Sede de Investigación Universitaria (SIU), Universidad de Antioquia, Medellín, Colombia.

Teléfono: (574) 219 6521; fax: (574) 2196565

nicolas.jaramillo@siu.udea.edu.co
}

Recibido: 11/12/07; aceptado:13/05/08
$B$ y A. (Nys.) strodei Root $(2,8)$. Tales dificultades se deben, principalmente, a que estas especies presentan una alta variabilidad fenotípica en los caracteres morfológicos utilizados en las claves y a la presencia de nuevas especies sin describir.

Otras metodologías utilizadas para diferenciar hembras del subgénero Nyssorhynchus son la identificación por cromosomas politénicos $(9,10)$, por isoenzimas $(11,12)$ y por técnicas de genética molecular $(13,14)$. Todas ellas contribuyen a esclarecer la identidad de los mosquitos Anopheles pero presentan el inconveniente de necesitar equipos y reactivos costosos, tiempo y experiencia para la interpretación de los datos, por lo cual son poco prácticas para su utilización en las actividades de rutina de los programas de control de malaria a nivel local. Por esta razón, se hace necesario explorar otras alternativas que en menos tiempo y con menos costos permitan, con un alto grado de confianza, asignar los especímenes capturados en el campo a su especie correspondiente.

La morfometría geométrica capta la mayor cantidad de información biológica en un menor número de variables tomadas sobre la estructura anatómica que se va a estudiar, lo cual resulta en un mayor poder estadístico para evaluar visual y analíticamente las diferencias de conformación de las estructuras biológicas (15). Esta técnica ha demostrado su utilidad para resolver problemas taxonómicos (16-18), logrando discriminar los integrantes de una especie en particular de otros que no lo son. Además, puede ser empleada para asignar un individuo desconocido a su grupo o especie más probable $(19,20)$.

Calle et al. (21), Delgado y Rubio-Palis (22) y RubioPalis $(23,24)$ utilizan morfometría "tradicional" 
(aplicación de análisis estadísticos univariados y multivariados a matrices de distancias entre puntos anatómicos) para identificar con buenos resultados algunas especies de Anopheles del subgénero Nyssorhynchus presentes en Colombia y Venezuela. Sin embargo, la morfometría tradicional tiene menor poder estadístico que la geométrica debido a que no logra, entre otras cosas, preservar la información espacial de los puntos anatómicos a través de los análisis (15). En Diptera la morfometría geométrica se ha utilizado para estudiar la variabilidad de las cepas de laboratorio de Aedes aegypti y la influencia de la densidad larvaria y las diferentes dietas sobre la arquitectura de las alas de esta misma especie $(25,26)$; pero no conocemos de trabajos publicados en este orden con la intención de apoyar la taxonomía.

El propósito de este trabajo fue el de utilizar la morfometría geométrica para identificar las especies de hembras de Anopheles del subgénero Nyssorhynchus presentes en cinco departamentos del norte, sur, centro y occidente de Colombia, lo cual es representativo del área de distribución del subgénero en el país.

\section{Materiales y métodos}

\section{Recolección y taxonomía de Anopheles}

El estudio morfométrico se hizo con 336 mosquitos hembras pertenecientes a 11 especies del subgénero Nyssorhynchus, tres correspondientes a la sección Argyritarsis y ocho a la sección Albimanus.

Los especímenes capturados cuando "aterrizaban" sobre el cebo humano protegido o las hembras recién emergidas procedentes de series 0 isofamilias $(22,23)$ se identificaron usando las claves morfológicas disponibles $(2,5,6)$.

Las especies identificadas a partir de hembras silvestres recolectadas en el municipio de Cáceres, departamento de Antioquia (070 34' 58" $\mathrm{N} ; 7^{\circ} 21^{\prime} 07^{\prime \prime}$ W) fueron: $A$. triannulatus Neiva y Pinto (30 especímenes), A. marajoara Galvao y Damasceno (30 especímenes) y $A$. nuneztovari (30 especímenes). En el municipio de Santa Marta, departamento de Magdalena (11 ${ }^{\circ} 15^{\prime} 30^{\prime \prime} \mathrm{N} ; 73^{\circ}$ 33' 38" W) se capturó $A$. aquasalis Curry (30 especímenes); en Leticia, departamento de
Amazonas (0412' 55” N; 69 56' 26" W), a $A$. darlingi (33 especímenes), y en Nuquí, departamento de Chocó (05 $45^{\circ}$ ' 45" N; $77^{\circ} 16^{\prime}$ $15^{\prime \prime} \mathrm{W}$ ), a $A$. albimanus (30 especímenes).

A partir de series e isofamilias de hembras capturadas en Puerto Asís, departamento de Putumayo (00 30' 57" N; 76 29' 42" W) se obtuvieron hembras de: $A$. braziliensis Chagas (30 especímenes), $A$. oswaldoi (30 especímenes), $A$. benarrochi $B$ (33 especímenes), A. strodei (33 especímenes) y $A$. rangeli (30 especímenes).

Las especies $A$. nuneztovari, $A$ oswaldoi y $A$. benarrochi $\mathrm{B}$ fueron confirmadas por PCR-RFLP (Polymerase Chain Reaction-Restriction Fragment Length Polymorphism) del marcador de ADNr ITS-2 (27).

Todas las alas examinadas se encuentran en la colección entomológica del Grupo de Chagas de la Universidad de Antioquia. Las exuvias de larvas y pupas, al igual que el ADN, se encuentran en el laboratorio del PECET de la Universidad de Antioquia.

\section{Selección de puntos anatómicos}

Se retiró el ala derecha de todos los especímenes desde la unión de la porción basal articulada al tórax. Se montó en una lámina de vidrio portaobjetos y se fijó con un cubreobjetos recubriendo sus lados con pegante. Posteriormente, se tomaron fotografías con una cámara digital Nikon Coolpix 990 adaptada a un microscopio estereoscópico Nikon modelo SMZ-800.

Sobre las imágenes de las alas se seleccionaron doce puntos entre los límites de las manchas basales y subcostales y las intersecciones de las venas Cu 1 y 2 con el borde distal del ala. Estos puntos permitieron capturar la configuración geométrica de cada ala (figura 1).

\section{Morfometría geométrica}

Los puntos anatómicos seleccionados se convirtieron en coordenadas en un plano bidimensional mediante la ayuda del programa tpsDig (28). La matriz de coordenadas, que representaba las configuraciones geométricas de las alas, se procesó usando el análisis generalizado de Procrustes $(15,29,30)$. 


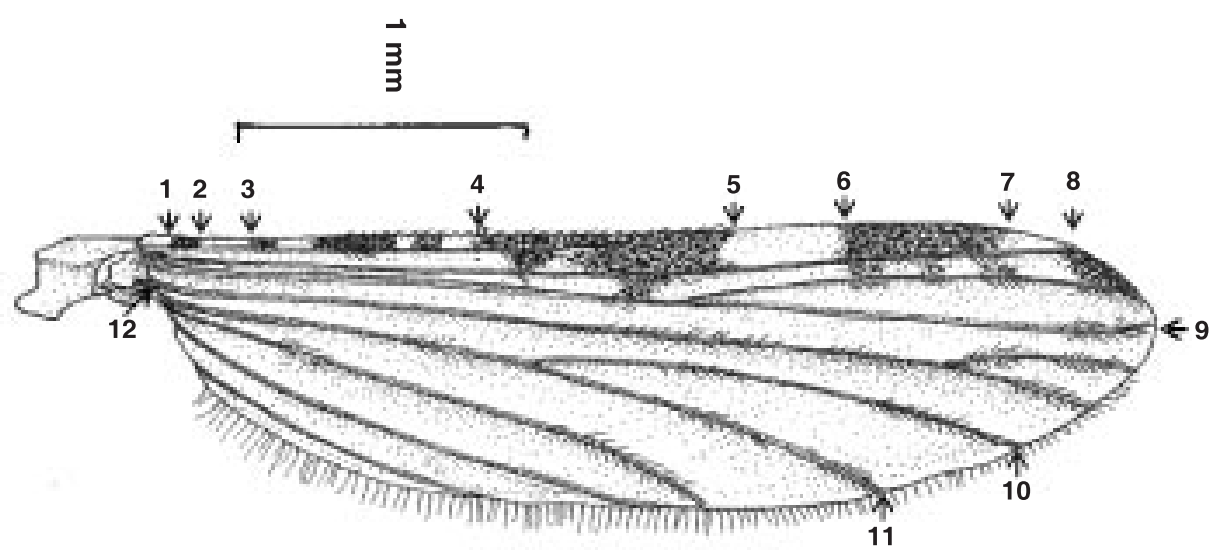

Figura 1. Puntos anatómicos. Localización de los puntos anatómicos tomados en el ala de los mosquitos hembra, (1) inicio de la mancha basal oscura (BD), (2) inicio de la mancha humeral clara (HP), (3) inicio de la mancha humeral oscura (HD), (4) inicio de la mancha subcostal oscura (SCD), (5) inicio de la mancha subcostal clara (SCP), (6) inicio de la mancha preapical oscura (PD), (7) inicio de la mancha preapical clara (PP), (8) finalización de la mancha apical clara (AP), (9) intersección de la vena R3 y el borde distal del ala, (10) intersección entre la vena Cu 1 y el borde distal del ala, (11) intersección entre la vena Cu 2 y el borde distal del ala y (12) intersección entre la vena plical y la vena cubital. La nomenclatura usada fue la propuesta por Faran en 1980 (3).

El procedimiento matemático del análisis generalizado de Procrustes se resume en tres pasos iterativos: 1) las configuraciones se ajustan a un tamaño único; 2) las configuraciones ajustadas se trasladan unas sobre otras de tal modo que coincidan sus centros de gravedad (centroides), y 3) se rotan hasta minimizar las distancias entre los puntos correspondientes, utilizando el criterio matemático de los cuadrados mínimos. Cada vez que se ejecutan estos tres pasos se calcula una configuración geométrica de referencia que, progresivamente, se va precisando con cada iteración. Al final del análisis generalizado de Procrustes, se obtienen variables que incorporan las desviaciones de cada configuración respecto a la de referencia. Estas variables son libres de la variación que no es biológica y no se ven afectadas por los cambios de tamaño, posición y orientación de las configuraciones en el espacio. Por lo tanto, contienen toda la información de la conformación del ala y se pueden utilizar para comparar las especies mediante análisis estadísticos multivariados.

El ajuste a un tamaño único (paso 1) se efectúa dividiendo todas las configuraciones por una variable de tamaño. Ésta se obtiene antes del análisis generalizado de Procrustes al calcular la raíz cuadrada de la suma de las distancias, elevadas al cuadrado, desde cada punto anatómico al centroide. Por tal motivo se le denomina tamaño-centroide y es un estimativo isométrico de la variación de tamaño de las muestras.

Los análisis se efectuaron con la ayuda de los programas computacionales MOGwin (19) y tpsRelw (31).

\section{Análisis del tamaño y la conformación}

El tamaño-centroide de cada especie fue utilizado para hacer las comparaciones de tamaño, mediante la prueba ANOVA. Los datos se analizaron utilizando el programa computacional JMP (32).

Las variables de conformación se utilizaron para realizar análisis discriminante. Las distancias de Mahalanobis, calculadas durante el análisis discriminante, se asociaron a la probabilidad de conocer qué tan alejada está cada conformación individual de la media de su especie o de la otra especie, lo que permite, de esta manera, hacer una reclasificación. 
Finalmente, se utilizaron 1.000 permutaciones para probar la hipótesis nula de que las distancias de Mahalanobis entre las especies y poblaciones eran producto del azar. La solidez de esta reclasificación se valoró con el estadístico kappa, el cual estima la concordancia entre dos clasificaciones, la dada por el análisis y una completamente aleatoria. La interpretación del valor estadístico fue sugerida por Landis y Koch (33). Las distancias de Mahalanobis también se utilizaron para evaluar las similitudes morfométricas por medio de la construcción de dendrogramas usando el método de neighbor joining.

Para el análisis discriminante, el cálculo de las distancias de Mahalanobis, su verificación estadística y la construcción de los dendrogramas se utilizaron los programas JMP (32), PADwin y el módulo neighbor del programa Phylip incluido en el PADwin (20).

La alometría se evaluó mediante análisis univariado de regresión lineal. Como variable dependiente se utilizó el primer factor canónico, calculado en el análisis discriminante, el cual representa la mayor porción de la variación de conformación derivada del análisis generalizado de Procrustes. El tamaño-centroide se utilizó como variable independiente de tamaño. Los valores significativos de regresión señalan un efecto del tamaño sobre la conformación (alometría). El coeficiente de determinación $\left(R^{2}\right)$ indica la porción de variación total que es explicada por el modelo. La porción de la variación total que no se ajusta al modelo indica la variación de conformación que no es el resultado colateral del crecimiento.

Los análisis fueron hechos con ayuda del programa computacional JMP (32).

\section{Visualización de las diferencias de conformación}

Se utilizó el programa computacional tpsReg (34) para obtener una representación visual de cada una de las conformaciones anatómicas promedio de cada especie. Éstas se observaron como deformaciones de rejillas respecto a la configuración promedio total (configuración de referencia) (32).

\section{Resultados}

\section{Subgénero Nyssorhynchus}

El análisis discriminante mostró que la conformación de las alas de las hembras del subgénero Nyssorhynchus es diferente entre las especies (lambda de Wilks=0,0013; gl: 230 y 2813; F: 12,92; $P=9,53 \times 10^{-125}$ ). La proyección de los individuos sobre los dos primeros factores canónicos, claramente diferencian a: $A$. nuneztovari, $A$. rangeli, $A$. darlingi, $A$. triannulatus y $A$. braziliensis, mientras que las otras cinco especies comparten áreas entre ellas y es más difícil distinguirlas individualmente (figura 2).

El análisis de conglomerado (cluster) realizado mediante neighbor joining arrojó cuatro grandes conglomerados de acuerdo con la conformación de las alas de las hembras (figura 3 ). El primer conglomerado estuvo conformado por $A$.

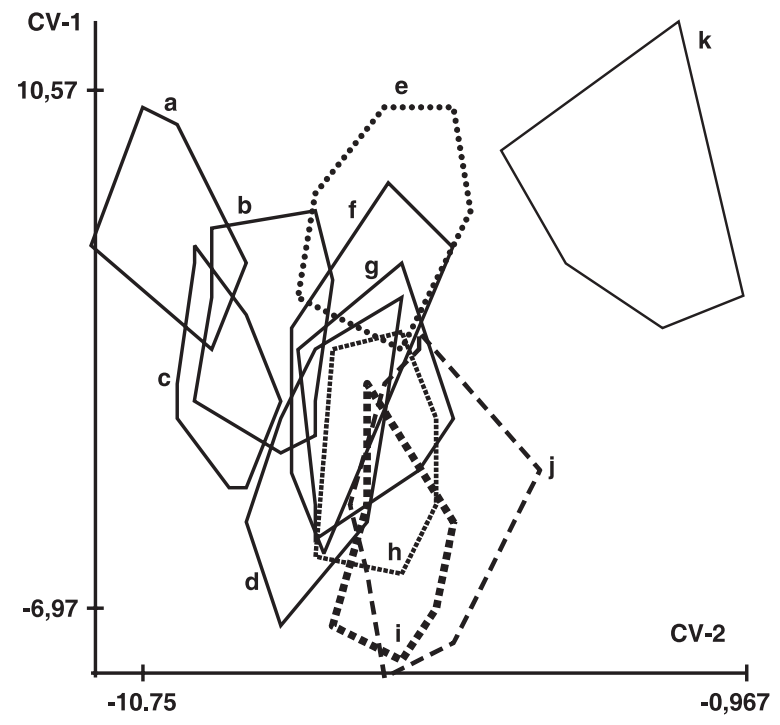

Figura 2. Distribución de 11 especies de Anopheles (Nyssorhynchus) correspondiente a su conformación del ala. Proyección de los individuos de cada especie sobre las variables canónicas $1(\mathrm{CV}-1)$ y 2 (CV-2) derivadas del análisis discriminante de la variación de conformación. Los polígonos delimitan áreas que agrupan a todos los individuos de una misma especie proyectados sobre el espacio multivariado de la conformación del ala. (a) A. darlingi; (b) A. braziliensis; (c) A., triannulatus; (d) A. marajoara; (e) A. nuneztovari; (f) A. albimanus; (g) $A$. aquasalis; (h) A. strodei; (i) $A$. benarrochi; (j) A. oswaldoi; (k) A. rangeli. 


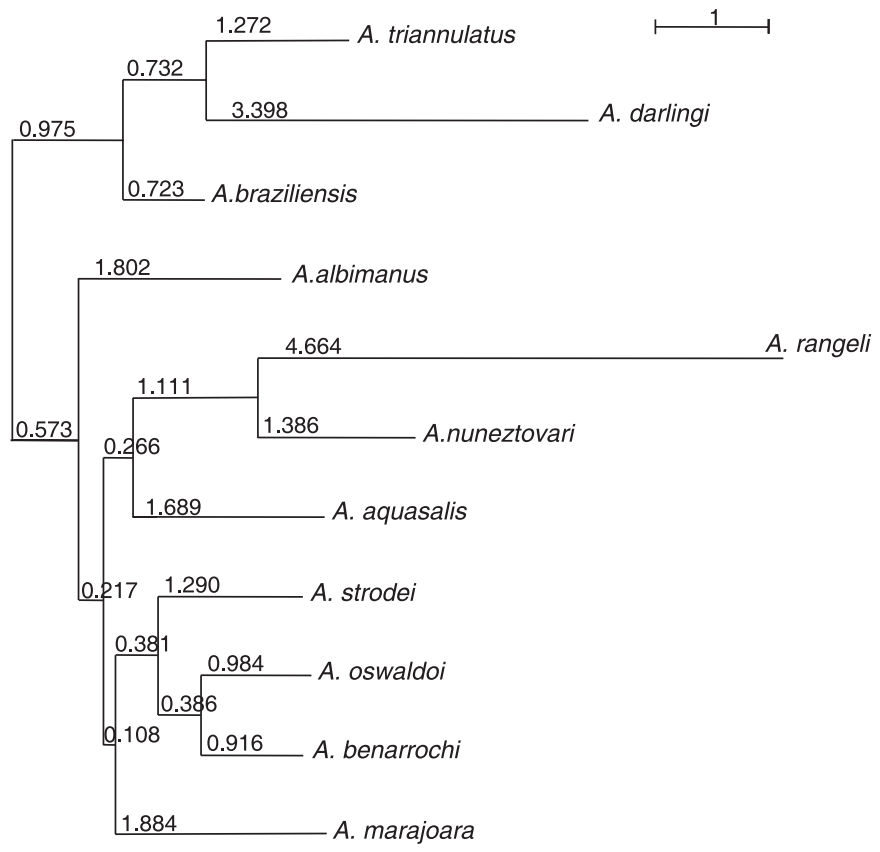

Figura 3. Dendrograma del subgénero Nyssorhynchus. Dendrograma sin raíz construido por el método de neighbor joining. Para construirlo se utilizaron las distancias de Mahalanobis entre las conformaciones promedio de las alas de las especies.

triannulatus, A. braziliensisy A. darlingi; el segundo, sólo por $A$. albimanus; el tercero, por $A$. rangeli, $A$. nuneztovari y $A$. aquasalis, y el cuarto, por $A$. strodei, A. oswaldoi, A. benarrochi B y $A$. marajoara (figura 3).

Las distancias de Mahalanobis calculadas entre cada par de especies fueron todas significativas al hacer la evaluación de la hipótesis nula mediante 1.000 permutaciones.

Para examinar en mayor detalle la variación morfométrica entre las especies se examinaron por separado los integrantes de las secciones Argyritarsis y Albimanus.

\section{Sección Argyritarsis}

Se encontraron diferencias significativas en el tamaño entre las tres especies de la sección Argyritarsis ( $\mathrm{F}=38,21 ; \mathrm{R}^{2}=0,45 ; \mathrm{gl}: 91 ; \mathrm{P}=9,69 \mathrm{x}$ $\left.10^{-13}\right)$. La especie más pequeña fue $A$. braziliensis y la más grande, $A$. darlingi (figura 4A).

El análisis discriminante demostró que la conformación de las alas de las hembras de la sección Argyritarsis es diferente entre las especies (lambda de Wilks=0,052; gl=40 y 142; $F=11,90$; $\mathrm{P}=1,64 \times 10^{-27}$ ) (figura 4B).

Las distancias de Mahalanobis demostraron que las conformaciones de cada especie son totalmente diferentes, y las permutaciones confirmaron que el resultado no fue producto del azar $(\mathrm{P}<0,0001)$.

Los individuos de $A$. marajoara se reclasificaron correctamente en el $100 \%$, mientras que $A$. darlingi y $A$. braziliensis lo hicieron con el $97 \%$. Según los criterios de Landis y Koch (33), la concordancia de la reclasificación fue casi perfecta (kappa $=0,97$ ).

Las diferencias entre especies fueron acentuadas, principalmente para $A$. darlingi la posición relativa de los puntos 1, 2 (representan la mancha basal oscura) con un alargamiento de esta mancha; mientras que los puntos 5 y 6 , que separan la mancha subcostal clara, se acercaron entre sí. En conjunto, tales movimientos generaron un ensanchamiento del ala. Por el contrario, en $A$. 


\section{Conformación}

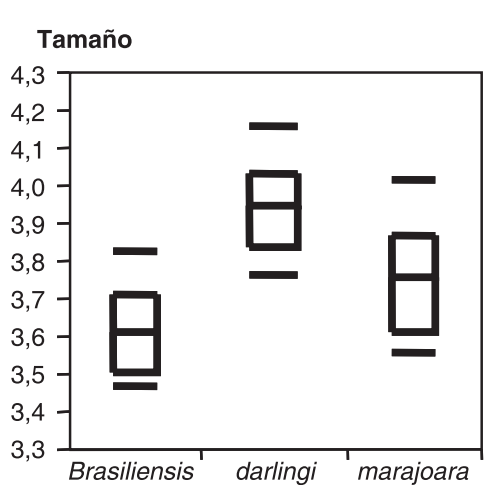

A

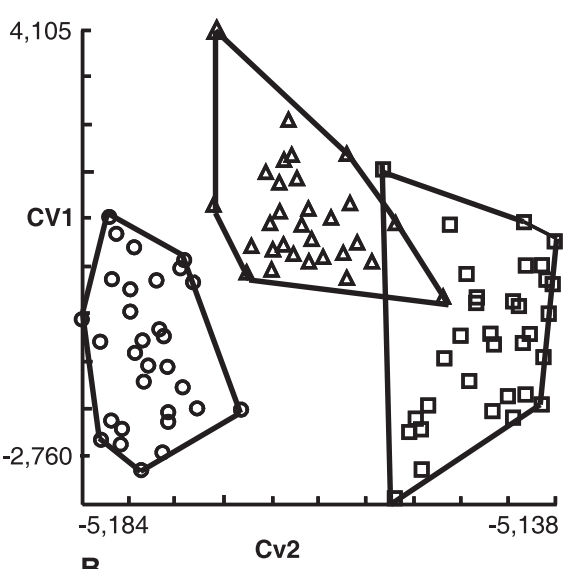

B

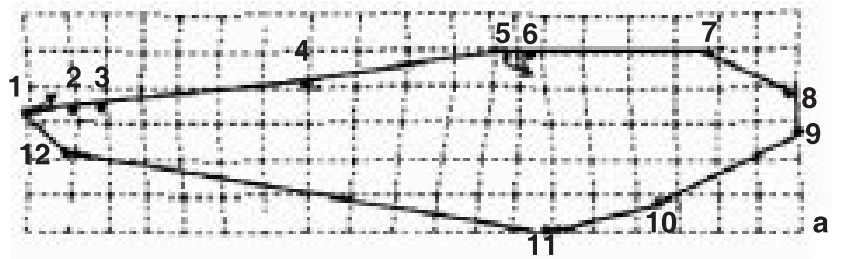

C

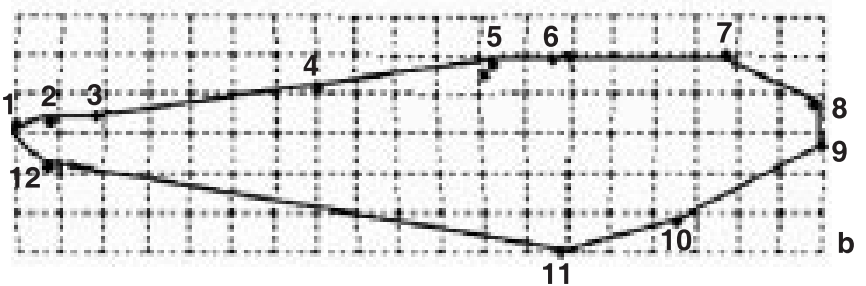

Figura 4. Representación gráfica de la diferenciación de las especies de la sección Argyritarsis. A. Las cajas esquemáticas resumen gráficamente la distribución de los individuos de cada especie de acuerdo con el tamaño. Cada caja muestra la mediana como la línea que atraviesa la mitad de la caja y los cuartiles 25 y 75 a los extremos. Los percentiles 10 y 90 se muestran como líneas sobre las cajas y debajo de ellas. B. Proyección de los individuos de cada especie sobre las variables canónicas 1 (CV-1) y 2 (CV-2) derivadas del análisis discriminante de la variación de conformación. Los polígonos delimitan áreas que agrupan a todos los individuos de una misma especie proyectados sobre el espacio multivariado de la conformación del ala. $A$. braziliensis $(\Delta)$, A. darlingi $(\square)$ y $A$. marajoara (o). C. La deformación de las rejillas permite visualizar el desplazamiento de la conformación del ala respecto a la configuración consenso de las especies de la sección Argyritarsis. (a) $A$. darlingi, (b) A. marajoara. Las flechas indican la magnitud y el sentido de desplazamiento de los puntos anatómicos.

braziliensisy A. marajoara se observó el movimiento inverso, lo cual provoca un estrechamiento del ala (figura 4C).

El análisis de regresión lineal fue significativo $\left(F=18 ; R^{2}=0,16 ; g l=92 ; P=0,0016\right)$, lo cual indica que la relación entre el tamaño y la conformación es significativa. El 16\% de la variación del modelo se puede explicar por la relación entre el tamaño y la conformación.

\section{Sección Albimanus}

Hubo diferencia de tamaño entre las especies $\left(\mathrm{F}=59,6 ; \mathrm{R}^{2}=0,63 ; \mathrm{gl}=181 ; \mathrm{P}=2,84 \times 10^{-48}\right)$, y $A$. triannulatus se destacó como la especie más pequeña, mientras que $A$. oswaldoi y $A$. rangeli fueron las más grandes (figura 5A).

El análisis discriminante demostró que $A$. rangeli y $A$. triannulatus se encontraban muy separadas de las otras seis especies (figura 5B). El 
MANOVA fue estadísticamente significativo (lambda de Wilks=0,99; gl=14 y 140; $P=9,22 \times$ $10^{-186}$ ). Las distancias de Mahalanobis demostraron que las conformaciones de cada especie son diferentes, y las permutaciones confirmaron que el resultado no fue producto del azar $(\mathrm{P}<0,0001)$.

Por medio del análisis discriminante, los individuos de $A$. rangeli y $A$. triannulatus se lograron reclasificar correctamente en el $100 \%$. A. albimanus, $A$. aquasalis y $A$. nuneztovari se reclasificaron en el $84 \%, 87 \%$ y $90 \%$, respectivamente. Mientras que $A$. strodei, $A$. oswaldoi y $A$. benarrochi $B$ se reclasificaron en el $85 \%, 76 \%$ y $70 \%$, respectivamente. Según los criterios de Landis y Koch (33), la concordancia general de la reclasificación fue casi perfecta (kappa $=0,85)$ (cuadro 1).

Los puntos anatómicos 1 y 2 que separan la mancha basal oscura, y los puntos 5 y 6 que separan la mancha clara subcostal, fueron los que presentaron mayor movimiento. Se observó que A. rangeli presentó una mayor separación de los puntos 5 y 6 y un acercamiento de los puntos 1 y 2 , lo que causa una deformación de esta región, con respecto a $A$. triannulatus que sufrió el efecto inverso sobre los mismos puntos (figura $5 \mathrm{C}$ ).

El análisis de regresión lineal fue significativo $\left(F=54,8 ; R^{2}=0,19 ; g l=242 ; P=4,55 \times 10^{-8}\right)$, lo cual indica que la relación entre el tamaño y la conformación es significativa. El 19\% de la variación del modelo se puede explicar por la relación entre el tamaño y la conformación.

A. oswaldoi, A. benarrochi By A. strodei se encuentran frecuentemente en simpatría en la región de Putumayo y, al utilizar las claves existentes, se presentan dificultades para su determinación taxonómica cuando se cuenta sólo con hembras. Por esta razón, se realizó el análisis sólo con estas especies, y se presentaron diferencias significativas entre sí en el tamaño promedio de sus alas $\left(F=23,99 ; R^{2}=0,34 ; g l=92 ; P=8,544 \times 10^{-09}\right.$ ) (figura 6A). Las distancias de Mahalanobis demostraron que las conformaciones de cada especie son totalmente diferentes $(P<0,0001)$.

El análisis discriminante sobre la variación de la conformación separó con un altísimo nivel de significancia a $A$. benarrochi $B, A$. oswaldoi y $A$. strodei (lambda de Wilks $=0,25 ; \mathrm{gl}=40$ y 142 ; $\mathrm{F}=5,08 ; \mathrm{P}=3,6 \times 10^{-08}$ ) (figura $6 \mathrm{~B}$ ). Al proyectar los individuos sobre los dos primeros factores canónicos, derivados del análisis discriminante de la variación de conformación de las alas, se presentó una superposición menor del $10 \%$ entre los polígonos correspondientes a $A$. benarrochi $B$ y $A$. oswaldoi, mientras que los individuos pertenecientes a $A$. strodei formaron un polígono aparte (figura 6B). El $90 \%$ de los individuos de $A$. oswaldoi y $A$. strodei y el $87 \%$ de $A$. benarrochi $B$ se reclasificaron correctamente en sus especies. Según los criterios de Landis y Koch (33), la concordancia de la reclasificación fue casi perfecta (kappa=0,88). Se observó un ensanchamiento del ala para $A$. oswaldoi y $A$. benarrochi $B$ al producirse un desplazamiento en la misma dirección de los puntos 3 (final de la mancha humeral clara), 4 (final de la mancha basal oscura), 5 y 6 (mancha subcostal clara), 7 y 8 (mancha preapical clara) y 11 (punto de intersección de la vena $\mathrm{Cu} 2$ y el borde del ala). Este desplazamiento fue de mayor magnitud para $A$. benarrochi $B$. En $A$. strodei se desplazaron los mismos puntos de las especies anteriores pero hacia el interior de la lámina del ala, lo que provoca un alargamiento y estrechamiento del ala (figura 6C).

El análisis de regresión lineal fue significativo $\left(\mathrm{F}=4,8 ; \mathrm{R}^{2}=0,27 ; \mathrm{gl}=92 ; \mathrm{P}=7,2 \times 10^{-06}\right)$, lo cual indica que la relación entre tamaño y conformación es significativa. El $27 \%$ de la variación del modelo se puede explicar por la relación entre el tamaño y la conformación.

\section{Discusión}

Las manchas de la vena costal del ala son caracteres utilizados en las claves para la determinación taxonómica de las hembras del subgénero Nyssorhynchus, especialmente la basal oscura, la humeral clara y la subcostal clara y oscura (5). En el presente estudio, también fueron importantes al utilizar la morfometría geométrica. Al incluir los extremos de tales manchas en los puntos anatómicos que definieron la configuración geométrica del ala se obtuvo la conformación propia de cada especie, y se logró separarlas entre sí con un alto grado de confianza. Además, se 


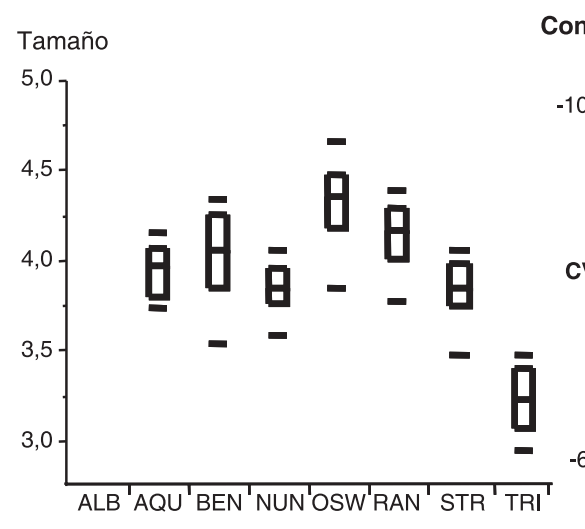

A

Conformación
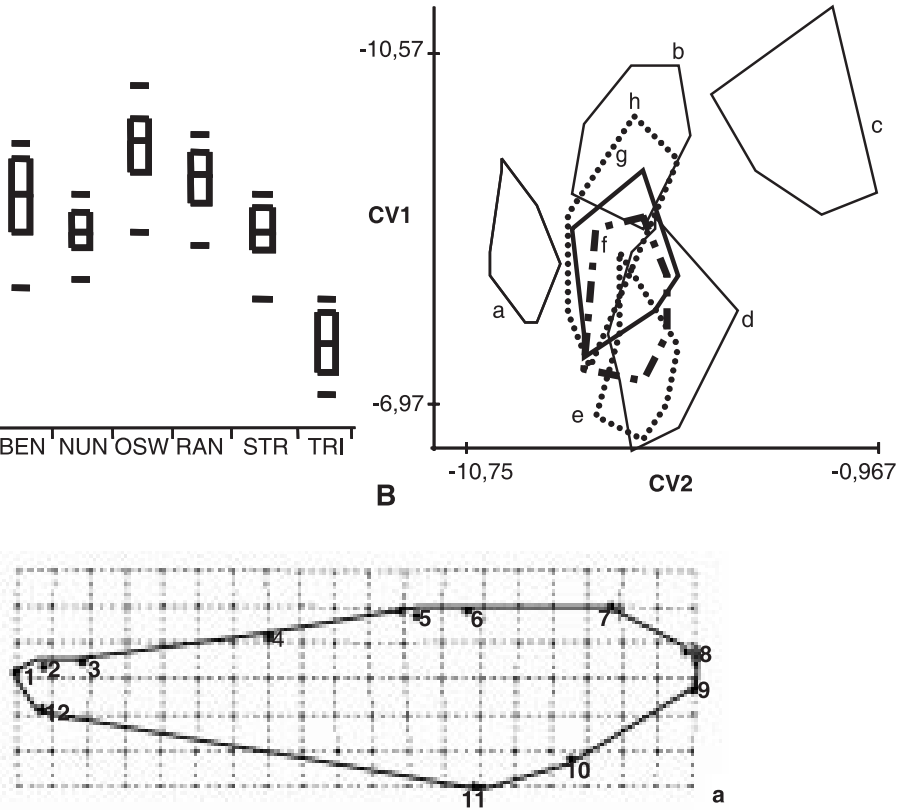

C

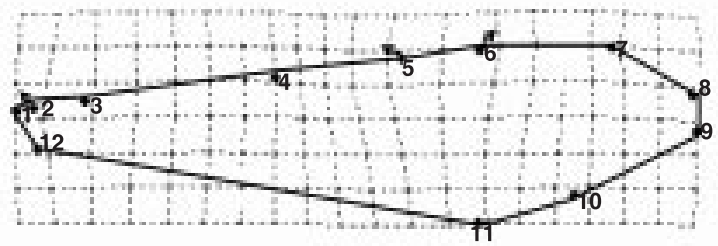

Figura 5. Representación gráfica de la diferenciación de las especies de la sección Albimanus. A. Las cajas esquemáticas resumen gráficamente la distribución de los individuos de cada especie de acuerdo con el tamaño. $A$. albimanus (ALB), $A$. aquasalis (AQU), A. benarrochi B (BEN), A. braziliensis (BRA), A. darlingi (DAR), A. marajoara (MAR), A. nuneztovari (NUN), A. oswaldoi (OSW), A. rangeli (RAN), A. strodei (STR) y A. triannulatus (TRI). B. Distribución de las especies a lo largo de las variables canónicas 1 (CV-1) y $2(\mathrm{CV}-2)$, derivadas del análisis discriminante de la variación de conformación. (a) A. triannulatus, (b) A. nuneztovari, (c) A. rangeli, (d) A. albimanus, (e) A. benarrochi B, (f) A. oswaldoi, (g) A. strodei, (h) A. aquasalis.C. La deformación de las rejillas permite visualizar el desplazamiento promedio de la conformación del ala de (a) A. triannulatus y (b) $A$. rangeli respecto a la configuración consenso de las especies del subgrupo Oswaldoi. Las flechas indican la magnitud y el sentido de desplazamiento de los puntos anatómicos.

pudo visualizar las diferencias de conformación alar entre las especies.

A partir de las distancias de Mahalanobis se demuestra que las 11 especies recolectadas en las diferentes regiones de Colombia son distintas y que estos resultados no son productos del azar. Además, aunque algunos individuos comparten áreas de intersección entre varias especies, los centroides (medias multivariadas de la conformación) de cada entidad permiten diferenciar las especies claramente. Se demuestra así la importancia de la conformación del ala para separar las especies del subgénero Nyssorhynchus.

El dendrograma obtenido a partir de las distancias de Mahalanobis del subgénero Nyssorhynchus (figura 3) muestra las relaciones de similitud morfométrica entre las especies. El dendrograma no coincide con las divisiones establecidas en secciones, ni tampoco coincide exactamente con las agrupaciones filogenéticas propuestas por 
Cuadro 1. Porcentajes de reclasificación correcta de las especies de la sección Albimanus. Los porcentajes de reclasificación correcta se muestran en negrilla en la diagonal. Los valores fuera de la diagonal señalan las reclasificaciones incorrectas. La reclasificación resultó de las distancias de Mahalanobis obtenidas durante el análisis discriminante de las conformaciones promedio de las alas.

\begin{tabular}{|c|c|c|c|c|c|c|c|c|c|}
\hline & & \multicolumn{6}{|c|}{ Especies } & \multirow[b]{2}{*}{ STR } & \multirow[b]{2}{*}{ TRI } \\
\hline & & ALB & AQU & BEN & NUN & osw & RAN & & \\
\hline \multirow[t]{8}{*}{ Especie predicha } & ALB & $84 \%$ & & $3.3 \%$ & $3.3 \%$ & $3 \%$ & & & \\
\hline & AQU & & $87 \%$ & $6.5 \%$ & $3.3 \%$ & $6 \%$ & & & \\
\hline & BEN & $6.5 \%$ & & $70 \%$ & $3.3 \%$ & $14 \%$ & & $6 \%$ & \\
\hline & NUN & $3.0 \%$ & $7 \%$ & & $90 \%$ & & & $3 \%$ & \\
\hline & OSW & $6.5 \%$ & $3 \%$ & $10 \%$ & & $77 \%$ & & $6 \%$ & \\
\hline & RAN & & & & & & $100 \%$ & & \\
\hline & STR & & $3 \%$ & $10 \%$ & & & & $85 \%$ & \\
\hline & TRI & & & & & & & & $100 \%$ \\
\hline
\end{tabular}

A. albimanus (ALB), A. aquasalis (AQU), A. benarrochi B (BEN), A. nuneztovari (NUN), A. oswaldoi (OSW), A. rangeli (RAN), A. strodei (STR), A. triannulatus (TRI).

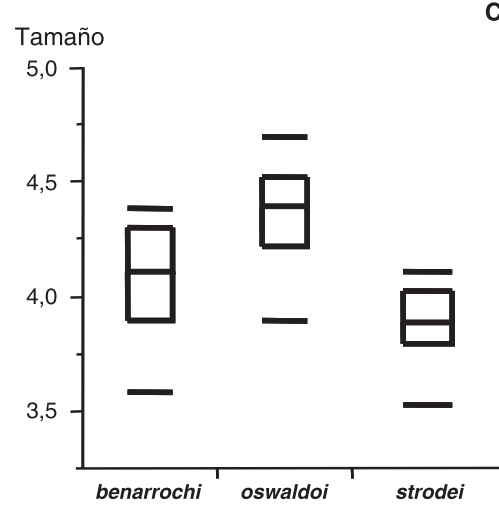

A

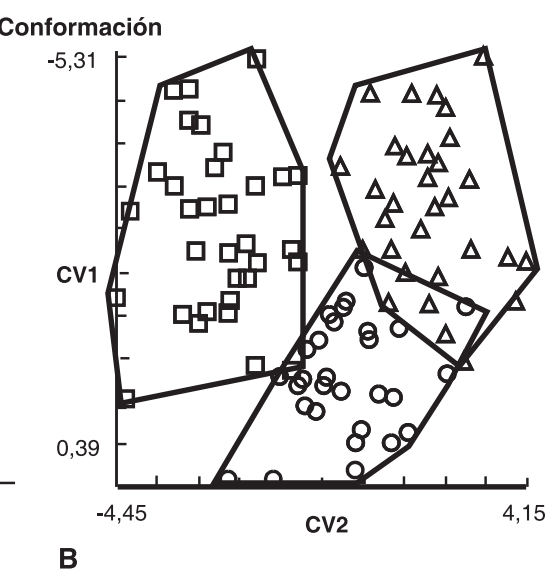

B

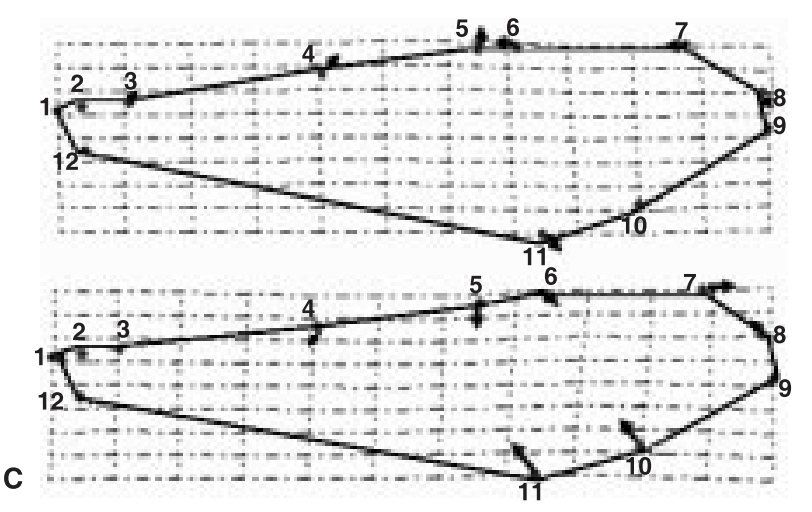

Figura 6. Representación gráfica de la diferenciación de las especies de difícil discriminación. A. Las cajas esquemáticas resumen gráficamente la distribución de los individuos de cada especie de acuerdo con el tamaño. B. Proyección de los individuos de cada especie sobre las variables canónicas 1 (CV-1) y 2 (CV-2) derivadas del análisis discriminante de la variación de conformación. $A$. benarrochi $B(0)$, A. strodei $(\square)$ y $A$. oswaldoi $(\Delta)$. C. La deformación de las rejillas permite visualizar el desplazamiento de la conformación del ala de (a) $A$. benarrochi $\mathrm{B}$ y (b) $A$. strodei respecto a la configuración consenso de las tres especies. Las flechas indican la magnitud y el sentido de desplazamiento de los puntos anatómicos. 
Faran y Linthicum (5). Sin embargo, algunas relaciones fenéticas propuestas desde la conformación de las alas muestran correlación con la hipótesis filogenética (5), como es el caso para A. oswaldoi, A. strodei, A. albimanus, $A$. nuneztovari y su especie hermana $A$. rangeli.

La posición de $A$. aquasalis cercano a $A$. nuneztovari y $A$. rangeli no es la esperada; esa especie se encuentra en una rama aparte, según Faran (2). Sin embargo, en algunos casos hay correspondencia entre la similitud morfométrica de estas especies y las relaciones estre especies medidas por diferentes marcadores moleculares. Tal es el caso de $A$. aquasalis y $A$. nuneztovari cuya cercanía en árboles reconciliados exhibe una fortaleza (medida por bootstrap) de $76 \%$ al utilizar los marcadores ribosómicos ADNr (18S y 28S) y mitocondriales ADNm (COI y COII) (35). Esta posición también coincide con la obtenida en la topología de los árboles creados por medio de los algoritmos de máxima parsimonia y máxima verosimilitud con soporte de bootstrap del $100 \%$, a partir de la información obtenida de los marcadores ribosómicos ITS 2 y mitocondriales COII (35).

La posición de $A$. triannulatus tampoco concuerda desde la división nominal de las secciones, aunque su cercanía a $A$. darlingi es viable teniendo en cuenta características morfológicas del adulto hembra como las escamas en el mesanepímero superior y las manchas basal y subcostal clara en el ala (2). La relación morfométrica es respaldada en el árbol construido a partir de la información de isoenzimas de cinco especies del subgénero Nyssorhynchus usando el algoritmo de UPGMA (36).

La cercanía de $A$. oswaldoi con $A$. benarrochi B no se puede comparar en otros árboles debido a que esta última especie se reconoció recientemente (27). Su cercanía se debe a que son muy similares morfológicamente, lo que impide su separación en el estadio de adulto hembra. Más adelante se analiza en mayor detalle las relaciones morfométricas entre estas especies.

La presencia de $A$. marajoara no concuerda con lo reportado por Faran (2) debido a que él separa las especies en dos secciones por la presencia o ausencia de un anillo en el tarsómero posterior cinco. La posición de esta especie cercana a las integrantes de la sección Albimanus coincide con el resultado de Sallum et al. (37), quienes utilizaron caracteres morfológicos de cinco especies de la sección Albimanus y cuatro de la Argyritarsis.

Entrando en el análisis morfométrico de la sección Argyritarsis, observamos que el tamaño puede reflejar diferencias taxonómicas entre las especies. Sin embargo, también puede ser el resultado de la combinación genética particular heredada de los padres y del crecimiento individual (factores maternos y efectos ambientales durante la ontogenia). Dado que las especies de esta sección no proceden de lugares donde habitan en simpatría, es necesario evaluar otras poblaciones para corroborar que este atributo biológico se mantiene a través de su distribución en el país y que puede ser empleado como un carácter discriminante.

Al seguir la clave de Faran y Linthicum (5), las hembras de $A$. darlingipresentan características morfológicas que permiten su diferenciación de las otras especies de la sección Argyritarsis. Sin embargo, $A$. braziliensis sólo se diferencia de $A$. marajoara en la presencia o ausencia de tufos en el segundo segmento abdominal y la coloración de las escamas en el octavo segmento abdominal. La presencia de tufos requiere un manejo cuidadoso de los especímenes para no perderlos y la coloración de las escamas, blanca o amarilla, depende de la fuente de luz, de la apreciación visual y la experiencia del taxónomo. Otras características como la proporción del área basal oscura del tarsómero posterior 2, la mancha y el número de manchas en la vena $\mathrm{R} 3$ del ala se mencionan en la clave para Anopheles de Colombia de González y Carrejo (38); sin embargo, tales características se superponen entre estas dos especies.

Por otro lado, por medio del análisis morfométrico de la conformación del ala, se logró reclasificar en el $97 \%$ los especímenes de cada especie (cuadro 1), lo que demuestra una estupenda correlación entre los resultados obtenidos por las claves existentes y la morfometría geométrica. 
Al visualizar la conformación promedio del ala de las especies (figura 4C), se observaron alas anchas en $A$. darlingi, y angostas en $A$. braziliensis y $A$. marajoara debido al movimiento hacia el interior del punto 11 (intersección entre la vena $\mathrm{Cu} 2$ y el borde distal del ala). Al saber que en la alometría sólo el 16\% del tamaño es aportado a la conformación, se deduce que la mayor porción de las diferencias (84\%) son propias de las poblaciones, lo cual refleja probablemente sus acervos genéticos diferentes.

Con respecto a la sección Albimanus, $A$. triannulatus exhibió las alas más pequeñas. Este resultado concuerda con otros reportes $(2,38)$ que sugieren que el tamaño de las alas puede ser utilizado como apoyo taxonómico para diferenciarla de las especies de la sección. Además, dentro de la clasificación nominal del subgénero Nyssorhynchus la separa como el subgrupo Triannulatus, debido, entre otros, a diferencias, en el estadio de hembra, con un parche conspicuo de escamas blancas o plateadas en el mesanepímero superior y escasa presencia de escamas blancas en el cuarto palpómero. Separación que concuerda con el hecho de que esta especie presentó una conformación de ala muy diferente de las otras siete especies (figura $5 \mathrm{C}$ ), ubicándose distante de todas en el espacio de los factores canónicos creado por la conformación del ala. Igualmente, al visualizar la conformación promedio se observó la deformación más extrema respecto a la de referencia, con un acercamiento en los puntos de las manchas basal oscura y subcostal clara; las cuales también son útiles para su discriminación al utilizar las claves existentes.

A. albimanus se ubica dentro de la sección como el grupo Albimanus $(2,38)$, porque tres caracteres morfológicos en el adulto hembra permiten separarlo de las otras especies: el palpómero cuatro completamente negro, la ausencia de tufos en el segmento abdominal dos y el quinto tarsómero anterior totalmente oscuro. El análisis discriminante de la conformación de las alas logró reclasificar el $84 \%$ de sus especimenes, lo que demuestra su utilidad para determinar una hembra que haya perdido los caracteres morfológicos más importantes (cuadro 1). Aunque su discriminación no es tan clara en la gráfica de dispersión (figura
5B), en la cual parece mezclarse con $A$. rangeli, $A$. strodei y $A$. aquasalis, es importante anotar que la proyección de los individuos sobre los dos primeros factores canónicos representan el $75 \%$ de la variación de conformación, mientras que la discriminación del $84 \%$ se obtuvo con el $100 \%$ de las variables de conformación.

Con $A$. aquasalis ocurre una situación similar; en Colombia no se le tiene por una especie importante en la transmisión de malaria, a excepción de algunas áreas en el departamento de La Guajira. Sin embargo, es considerada el vector principal de malaria en Venezuela. Morfológicamente se diferencia de las otras especies del grupo Oswaldoi por poseer una mancha basal oscura en el tarsómero posterior dos de 0,4 a 0,55 la longitud del segmento. Pero las manchas características del ala son muy similares a las de $A$. nuneztovari, uno de los vectores más importantes en Colombia, lo cual puede llevar a identificaciones erróneas en ausencia de las patas posteriores. El análisis discriminante de la conformación del ala separó completamente estas dos especies y logró reclasificar correctamente a $A$. aquasalis en el $87 \%$, mientras que a $A$. nuneztovari en el $90 \%$ (cuadro 1), con lo que se demuestra de nuevo la utilidad de la morfometría geométrica para apoyar la taxonomía.

Por su parte, la conformación del ala de $A$. rangeli, fue la otra más diferente dentro de la sección Albimanus, representada por la separación de los puntos que constituyen la mancha subcostal clara, lo cual concuerda con la importancia de este carácter morfológico para diferenciar esta especie de otras de la sección. La posición de A. rangeli, separada del grupo Oswaldoi debido a su conformación del ala, no concuerda con lo propuesto por Faran (2), quien usa las características morfológicas de la larva, pupa y adulto para asignar esta especie dentro del grupo mencionado. Sin embargo, si sólo se tuvieran en cuenta las medidas del ala (mancha subcostal clara mayor de 0,5), $A$. rangeli se diferenciaría claramente de las demás del grupo, excepto con algunas hembras de $A$. nuneztovari morfotipo II.

La dificultad para diferenciar $A$. rangeli de $A$. nuneztovari ocurre frecuentemente con los 
individuos del morfotipo II de $A$. nuneztovari, el cual posee una proporción de 1,8 a 3,0 en la mancha humeral clara con respecto a la mancha basal oscura de la vena subcostal del ala (22) mientras que en $A$. rangeli mide de 1,8 a 3,5. Por lo tanto, es necesario usar la quetotaxia del cuarto estadio larvario, de la pupa o de la genitalia masculina para diferenciar correctamente estas dos especies (2).

En este trabajo el análisis discriminante de la conformación del ala de las hembras permitió diferenciar $A$. rangeli de $A$. nuneztovari, y se lograron porcentajes de reclasificación del $84 \%$ y el $90 \%$, respectivamente (cuadro 1). Se demuestra con estos resultados el potencial de la morfometría geométrica para discriminar estas dos especies que pertenecen a un grupo hermano y cuyas hembras poseen características morfológicas tan similares que al ser cuantificadas por los métodos tradicionales no permiten diferenciarlas. Sin embargo, se necesita contar con poblaciones que habiten en simpatría para corroborar esta afirmación.

La alometría demostró que para estas cinco especies sólo el $19 \%$ del tamaño es aportado a la conformación, de donde se deduce que la mayor porción de las diferencias (81\%) son propias de las poblaciones, lo cual probablemente es reflejo de sus acervos genéticos diferentes.

Dentro del grupo Oswaldoi, las hembras de $A$. benarrochi $B$ halladas en el departamento del Putumayo, Colombia, presentan características morfológicas que comparten en muchos casos con A. oswaldoi (27).

En este trabajo se demuestra que el tamaño del ala de estas dos especies y de $A$. strodeipermite separarlas claramente (figura $6 \mathrm{~A}$ ), carácter que se podría utilizar como apoyo a las claves morfológicas que se puedan proponer para diferenciarlas. La separación de estas tres especies también estuvo apoyada por la conformación del ala, la cual demostró que $A$. oswaldoi y $A$. benarrochi $B$ eran más parecidos entre sí, representados por alas anchas, y diferentes de $A$. strodei la cual tiene alas más angostas (figura $6 \mathrm{C}$ ). El análisis discriminante de la conformación del ala separó, sin lugar a dudas, ambas especies (figura 6B) y logró reclasificar correctamente el $87 \%$ de las hembras de $A$. benarrochi $B$ y el $90 \%$ de las de $A$. oswaldoi. Hasta la fecha, las hembras de $A$. oswaldoi y $A$. benarrochi $B$ sólo se pueden separar sin ambigüedades por técnicas de biología molecular, usando PCR-RFLP del ITS-2 (27). A pesar de que esta metodología separa ambas especies en el $100 \%$, la necesidad de conservar los ejemplares en sílica-gel y los altos costos de procesamiento dificultan su aplicación a gran escala.

Por otro lado, $A$. benarrochi $B$ no se puede diferenciar de $A$. strodei porque la proporción de la mancha basal oscura del tarsómero posterior dos con relación a la longitud del segmento se superpone entre ellas $(0,17-0,32$ y $0,25-0,35$, respectivamente). En el ala, la proporción de la mancha clara humeral de la vena subcostal con respecto a la mancha basal oscura no permite diferenciar las hembras de las especies mencionadas debido a que, de nuevo, hay superposición: 1,7-3,3 para $A$. benarrochi $B$ y $2,0-4,0$ para $A$. strodei. Igual sucede con la mancha subcostal clara con relación a la subcostal oscura, la cual presenta una proporción de $0,1-0,5$ para $A$. benarrochi $B$ y de $0,25-0,5$ para $A$. strodei. Al utilizar la morfometría geométrica, el análisis discriminante de la conformación del ala logró de nuevo separar ambas especies (figura 6B) y logró reclasificar correctamente las hembras de $A$. benarrochi $B$ en el $87 \%$ y las de $A$. strodei en el $90 \%$.

Debido a que estas tres especies habitan en simpatría y al hecho de que la conformación es la variable que más pesa en su discriminación (el efecto alométrico fue de sólo el $27 \%$ ) es muy probable que sus diferencias sean el reflejo de características genéticas y no el resultado de interacciones con el medio ambiente o de diferenciación geográfica.

En conclusión, la morfometría geométrica logró distinguir las hembras de 11 especies del subgénero Nyssorhynchus presentes en Colombia utilizando sólo la información contenida en la conformación del ala. Estos resultados permiten proponer la utilización de los puntos anatómicos seleccionados en este trabajo para procesarlos mediante el algoritmo generalizado de Procrustes, 
de tal manera que se apoye de manera sólida el trabajo taxonómico con las especies del subgénero Nyssorhynchus.

\section{Agradecimientos}

Por su colaboración en la captura de los Anopheles, agradecemos a Rafael Burbano y Eliécer Gualguan del Departamento Administrativo de Salud Putumayo (DASALUD). A William Galarza director de DASALUD por la colaboración con el personal de campo. A Iván Darío Vélez, director del Programa de Estudio y Control de Enfermedades Tropicales (PECET) de la Universidad de Antioquia. En especial a Manuela Herrera y Lorena Orjuela por su enorme colaboración en la consecución de especímenes. Este artículo esta dedicado a nuestros amigos Edison Correa, Carlos Andrés Pérez y Marcos Pérez, a quienes su devoción en la captura de mosquitos les costó la vida.

\section{Conflicto de interés}

Los autores declaramos que no existe un posible conflicto de intereses en este manuscrito.

\section{Financiación}

Este estudio fue financiado por el Comité para el Desarrollo de la Investigación (CODI), Universidad de Antioquia, código CPT 0214.

\section{Referencias}

1. World Health Organization. Malaria. Fact Sheet № 94. Geneve: WHO; 2007. [Consultado: marzo de 2008]. Disponible en: http://www.who.int/mediacentre/ factsheets/fs094/en/index.html.

2. Olano VA, Brochero LH, Saenz R, Quiñones ML, Molina JA. Mapas preliminares de la distribución de especies de Anopheles vectores de malaria en Colombia. Biomédica. 2001;21:402-8.

3. Quiñones ML, Ruiz F, Calle DA, Harbach RE, Erazo HF, Linton YM. Incrimination of Anopheles (Nyssorhynchus) rangeli and An. (Nys.) oswaldoi as natural vectors of Plasmodium vivax in Southern Colombia. Mem Inst Oswaldo Cruz. 2006;101:617-23.

4. Faran ME. Mosquito studies (Diptera: Culicidae). XXXIV. A revisions of the Albimanus Section of the subgenus Nyssorhynchus of Anopheles. Contrib Am Entomol Inst. 1980;15:1-215.

5. Faran ME, Linthicum KJ. A Handbook of the species of Anopheles (Nyssorhynchus) (Diptera: Culicidae). Mosq Syst. 1981;13:1-81.
6. Linthicum KJ. A revision of the Argyritarsis Section of the subgenus Nyssorhynchus of Anopheles (Diptera: Culicidae). Mosq Syst. 1988;20:99-271.

7. Suárez MF, Quiñones M, Fleming GA, Robayo M. Guía introductoria a la morfología de Anopheles y clave para determinación de las principales especies de Colombia. Bogotá, D.C.: Ministerio de Salud; 1988.

8. Quiñones ML, Harbach RE, Calle DA, Ruiz F, Erazo HF. Variante morfológica de Anopheles benarrochi (Diptera: Culicidae) en Putumayo, Colombia. Biomédica. 2001;21:351-9.

9. Frizzi G. Salivary gland chromosomes of Anopheles. Nature. 1947;160:226-8.

10. Frizzi G. Etude cytogenetique d'Anopheles maculipennis en Italie. Bull World Health Organ. 1953;9:335-44.

11. Miles SJ. A biochemical key to adult members of the Anopheles gambiae group of species (Diptera: Culicidae). J Med Entomol. 1979;15:297-9.

12. Foley DH, Bryan JH. Electrophoretic keys to identify members of the Anopheles punctulatus complex of vector mosquitoes in Papua New Guinea. Med Vet Entomol. 1993;7:49-53.

13. Wilkerson RC, Parsons TJ, Klein TA, Gaffigan TV, Bergo E, Consolim J. Diagnosis by random amplified polymorphic DNA polymerase chain reaction of four cryptic species related to Anopheles albitarsis from Paraguay, Argentina and Brazil. J Med Entomol. 1995; 32:697-704.

14. Marelli MT, Malafronte RS, Florez-Mendoza C, Lourenco- de- Oliveira R, Kloetzel JK, Marinotti 0 . Sequence analysis of the second internal transcribed spacer of ribosomal DNA in Anopheles oswaldoi (Diptera: Culicidae). J Med Entomol. 1999;36:679-84.

15. Rohlf FJ, Marcus LF. A revolution in morphometrics. Trends Ecol Evol. 1993;8:129-32.

16. Matias A, De la Riva JX, Torrez M, Dujardin JP. Rhodnius robustus in Bolivia identified by its wings. Mem Inst Oswaldo Cruz. 2001;96:947-50.

17. Villegas J, Feliciangeli MD, Dujardin JP. Wing shape divergence between Rhodnius prolixus from Cojedes (Venezuela) and Rhodnius robustus from Mérida (Venezuela). Infect Genet Evol. 2002;2:121-8.

18. Gumiel L, Catala S, Noireau F, Rojas de Arias, Garcia A, Dujardin JP. Wing geometry in Triatoma infestans (Klug) and T. melanosoma Martinez, Olmedo \& Carcavallo (Hemiptera:Reduvidae). Syst Entomol 2003;28:173-9.

19. Dujardin JP. MOGwin (Software for Generalized Procrustes Analysis). Institut de Recherches pour le Développement. 2004. [Consultado: marzo de 2008]. Disponible en: http://www.mpl.ird.fr/morphometrics/ mog/index.html 
20. Dujardin JP. PADwin (Software for Discriminant Analysis). Institut de Recherches pour le Développement. 2004. [Consultado: marzo de 2008]. Disponible en: http://www.mpl.ird.fr/morphometrics/pad/

21. Calle DA, Quiñones ML, Erazo H, Jaramillo 0 . Morphometric discrimination of females of five species of Anopheles of the subgenus Nyssorhynchus from Southern and Northwest Colombia. Mem Inst Oswaldo Cruz. 2002;97:1191-5.

22. Delgado N, Rubio-Palis Y. Identification of Anopheles (Nyssorhynchus) (Diptera: Culicidae) occurring in Western Venezuela. Mosq Syst. 1993;25:222-30.

23. Rubio-Palis Y. Caracterización morfométrica de poblaciones de Anopheles (Nyssorhynchus) darlingi del sur de Venezuela. Bol Entomol Venez. 1998;13: 141-72.

24. Rubio-Palis Y. Anopheles (Nyssorhynchus) de Venezuela: taxonomía, bionomía, ecología e importancia médica. Maracay: Escuela de Malariología y Saneamiento Ambiental; 2000. p. 120.

25. Jirakanjanakit N, Dujardin JP. Discrimination of Aedes aegypti (Diptera: Culicidae) laboratory lines based on wing geometry. Southeast Asian J Trop Med Public Health. 2005;36:858-61.

26. Jirakanjanakit $\mathbf{N}$, Leemingsawat $\mathbf{S}$, Thongrungkiat S, Apiwathnasor C, Singhaniyom S, Bellec C, et al. Influence of larval density or food variation on the geometry of the wing of Aedes (Stegomyia) aegypti. Trop Med Int Health. 2007;12:1354-60.

27. Ruiz F, Quiñones ML, Erazo H, Calle DA, Alzate FA, Linton YM. Molecular differentiation of Anopheles (Nyssorhynchus) benarrochi and A. (N.) oswaldoi from Southern Colombia. Mem Inst Oswaldo Cruz. 2005;100:155-60.

28. Rohlf J. tpsDig, version 1.04. Stony Brook, NY: Department of Ecology and Evolution, State University of New York at Stony Brook; 2004. [Consultado: marzo de 2008]. Disponible en: http://life.bio.sunysb.edu/ morph.

29. Rohlf FJ, Slice DE. Extensions of the Procrustes method for the optimal superimposition of landmarks. Syst Zool. 1990;39:40-59.
30. Bookstein FL. Morphometrics tools for landmark data: Geometry and biology. Cambridge: Cambridge University Press; 1991.p.435.

31. Rohlf J. tpsRelw, version 1.39. Stony Brook, NY: Department of Ecology and Evolution, State University of New York at Stony Brook; 2004. [Consultado: marzo de 2008]. Disponible en: http://life.bio.sunysb.edu/ morph.

32. SAS Institute Inc. JMP® Statistisc and Graphics Guide, version 3.1. Cary, NC: SAS Institute Inc; 1999.p.592.

33. Landis JR, Koch GG. The measurement of observer agreement for categorical data. Biometrics. 1977;33:159-74

34. Rohlf, J. tpsRegr, version 1.28. Stony Brook, NY: Department of Ecology and Evolution, State University of New York at Stony Brook; 2003. [Consultado: marzo de 2008]. Disponible en: http://life.bio.sunysb.edu/ morph.

35. Sallum M, Schultz T, Foster P, Arostein K, Wirtz R, Wilkerson C. Phylogeny of Anophelinae (Diptera: Culicidae) based of nuclear, ribosomal and mitochondrial DNA secuences. Syst Entomol. 2002; 27:361-82.

36. dos Santos JM, Maia J de F, Tadei WP, Rodríguez GA. Isoenzymatic Variability among five Anopheles species belonging to the Nyssorhynchus and Anopheles subgenera of the Amazon region, Brazil. Mem Inst Oswaldo Cruz. 2003;98:247-53.

37. Sallum MA, Bergo ES, Flores DC, Forattini OP. Systematic Studies on Anopheles galvaoi Causey, Deane \& Deane from the Subgenus Nysssorhynchus Blanchard (Diptera: Culicidae). Mem Inst Oswaldo Cruz. 2000;97:1177-89.

38. Gonzales R, Carrejo N. Introducción al estudio taxonómico de Anopheles de Colombia Claves y notas de distribución. Cali: Editorial Litocencoa; 2007. p. 237. 